\title{
A simulation of imaging capabilities for the Chinese Spectral Radioheliograph
}

\author{
Jing $\mathrm{Du}^{1}$, Yihua $\mathrm{Yan}^{1}$, and Wei Wang ${ }^{1}$
}

${ }^{1}$ Key Laboratory of Solar Activity, National Astronomical Observatories, Chinese Academy of Sciences, Beijing 100012, China.email: jdu@nao.cas.cn

Abstract. This work applied Chinese Spectral Radioheliograph(CSRH) to simulate the quiet Sun to produce the radio image. Then, we simulate a point source, and make all antennas have a typical phase error $\left(5^{\circ}\right)$ to calculate the dynamic range. It is found that the dynamic range agrees with the theoretical value. The image processing and simulated results are presented.

Keywords. Chinese Spectral Radioheliograph, image simulation, the dynamic range

\section{Introduction}

The Chinese Spectral Radioheliograph in centimetric-decimetric wave range is a solardedicated radio interferometric array that will be used to carry out imaging spectroscopy of the Sun, to produce high spatial resolution, high time resolution and high frequency resolution images of the Sun simultaneously (Yan. et al. 2009). Therefore, it is important to simulate the sources of different structures which based on several emission models to produce different images.

The CSRH array with 100 antennas is arranged on 3 spiral arms, and the maximum baseline length is $3 \mathrm{~km}$. The frequency range is $0.4-15 \mathrm{GHz}$, but the $\mathrm{RF}$ signal is divided into $0.4-2 \mathrm{GHz}(\mathrm{CSRH}-\mathrm{I})$ and $2-15 \mathrm{GHz}(\mathrm{CSRH}-\mathrm{II})$ bands. The CSRH-I contains 40 antennas of $4.5 \mathrm{~m}$, and the CSRH-II contains 60 antennas of $2 \mathrm{~m}$ (Yan. et al. 2009).

\section{Method and Results}

For a small field of view, the visibility function is a Fourier component of the true sky brightness distribution.
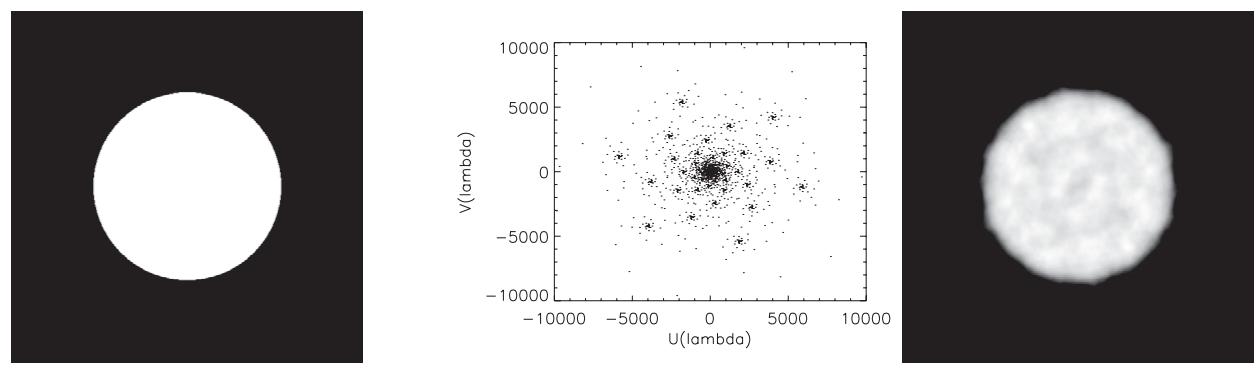

Figure 1. Left panel: The model of the quiet Sun. Middle panel: The u-v coverage of the CSRH-I at $1 \mathrm{GHz}$. Right panel: Full disk radio image of the model observed by CSRH-I array.

\subsection{Simulate the quiet Sun}

As above, we simulate a model of the quiet Sun which is with $512^{*} 512$ pixels. The corresponding field of view is $1^{\circ *} 1^{\circ}$ (Fig. 1 . Left panel). Then, we calculate the uv coverage of 
the CSRH-I array (Fig. 1. Middle panel). Since the spatial frequency plane(u, v) cannot fully sampled due to the limited numbers of antennas in an aperture synthesis array. Thus, the sampled visibilities can be obtained by the true visibilities multiplied by the sampling function. Hence, we can obtain the image which is the dirty map of the model of the quiet Sun by Fourier inversion of the sampled visibility function (Fig. 1. Right panel) (Taylor, et al. 1999, Napier, et al. 1983).

\subsection{Simulate a point source to calculate the dynamic range}

The dynamic range attained on an image depend on the type, size and distribution of errors in the measurements of the visibility. The visibility errors contain the baselinebased and the antenna-based errors of various magnitudes, in this report, we just consider the antenna-based errors. For the purpose of analysis, it is simplest to consider a point source-for which all visibilities are the same. So, we simulate a point source and assume it be observed with CSRH-I array to produce the dirty map (Fig. 2. Left panel). Then, we suppose that all antennas have a closure-phase error $\left(5^{\circ}\right)$ which is the Uniform distribution or the Gaussian distribution to calculate the dynamic range (Fig. 2. Middle panel). The
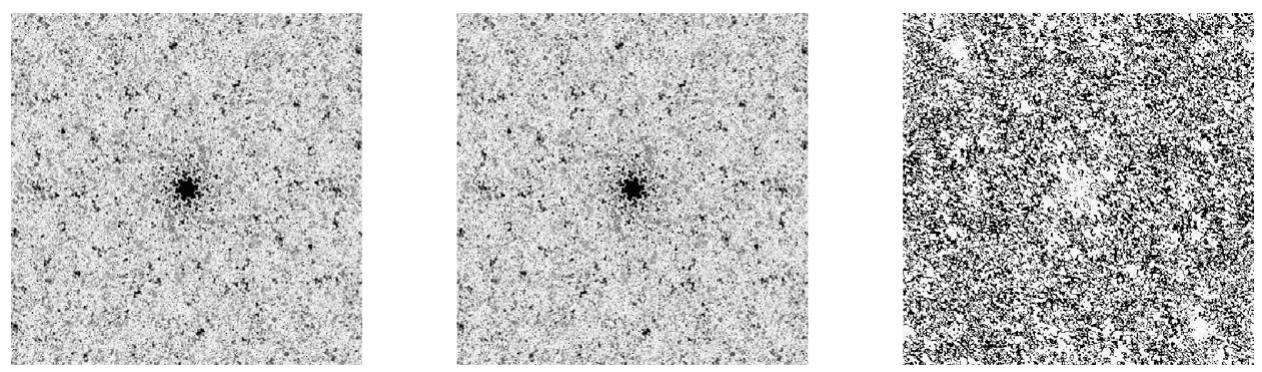

Figure 2. Left panel: The dirty image of the simulated point source which is observed by CSRH-I array. The region is $512^{*} 512$ pixels, the corresponding field of view is $1^{\circ *} 1^{\circ}$. Middle panel: The dirty map observed by CSRH-I array which is ignored the amplitude errors, and supposed that all antennas have a closure-phase error $\left(5^{\circ}\right)$ which is distributed uniformly. Right panel: The residual image between the two dirty images.

dynamic range of an image is defined as the ratio between the peak brightness of the strongest compact source and the standard deviation of the errors in the image (Koshiishi et al. 1994). The errors can be calculated by taking the differential image between the above two dirty images (Fig. 2. Right panel). Therefore, we can obtain the dynamic range of the point source is $31.89794 \mathrm{~dB}$ (the error is the Uniform distribution) or $26.6117 \mathrm{~dB}$ (the error is the Gaussian distribution). These are consistent with $D=\frac{1}{\phi} \sqrt{\frac{N(N-1)}{2}} \approx$ $\frac{N}{\sqrt{2} \phi}$ (N is the number of the antennas, $\phi$ is the phase error, $\mathrm{D}=25.0520 \mathrm{~dB}$ ) when all antennas have a typical phase error (Taylor et al. 1999).

\section{Conclusion}

(1)A good image of the model of the quiet Sun can be obtained with the CSRH-I array.

(2)The dynamic range which ignored the amplitude error and supposed all antennas have a $5^{\circ}$ phase error agrees with the theoretical value.

\section{References}

Koshiishi, H., Enome, S., Nakajima, H., et al. 1994, PASJ, 46, L33-L36

Napier, P. J., Thompson, A. R., \& Ekers, R. D. 1983, IEEE

Taylor, G. B., Carilli, C. L., \& Perley, R. A. 1999, ASP Conf. Ser. v. 6

Yan, Y. H., Zhang, J., Wang, W., et al. 2009, Earth Moon Planet, 97,100 